\title{
Comparison of the effect of acetazolamide tablets and Sustets on diurnal intraocular pressure in patients with chronic simple glaucoma
}

\author{
P W Joyce, K B Mills
}

\begin{abstract}
Twenty patients with primary open-angle glaucoma uncontrolled on single topical therapy completed a double dummy crossover study to compare acetazolamide tablets with a sustained release formulation (Sustet). The two preparations were equally effective, but no direct relationship was found between the intraocular pressure and the plasma concentration of acetazolamide. No difference between the formulations was found in the frequency or severity of side effects.
\end{abstract}

Acetazolamide (Diamox) is a carbonic anhydrase inhibitor used in ophthalmology to control intraocular pressure (IOP). ${ }^{1}$ It is prescribed in conjunction with single or multiple topical treatment, and has been available for many years in the UK as a $250 \mathrm{mg}$ tablet. In the early $1960 \mathrm{~s}$ a sustained release formation (Sustet) was marketed containing $500 \mathrm{mg}$ of the active drug. Several studies have confirmed the efficacy of both preparations in lowering IOP. ${ }^{2-8}$ However, many side effects have been encountered and were thought to be dose related with both formulations. ${ }^{910}$ In an effort to reduce these effects smaller doses of acetazolamide have been tried over short periods of time. ${ }^{112}$ However, no study has compared the effects of the same doses of the two formulations available in the UK on IOP over 12- or 24-hour dosage intervals.

Material and methods

As part of a study ${ }^{13}$ of the role of acetazolamide in glaucoma 35 patients with primary open-angle glaucoma were enrolled in a double dummy crossover study to compare a once or twice daily dose of acetazolamide tablets with the same dose of Sustet. The IOP of these patients was inadequately controlled on single topical treatment (pilocarpine/timolol). All patients fulfilled the following criteria: IOP $>22 \mathrm{mmHg}$, an open angle on gonioscopy, a glaucomatous field defect, and cupped optic discs. The exclusion criteria were previous intraocular surgery, ocular injury, ocular inflammation, and other types of glaucoma.

After giving written, informed consent each patient underwent a full physical and ocular examination. The latter included visual acuity (Snellen), visual fields (Goldmann), applanation tonometry, gonioscopy, and ophthalmoscopy. A blood sample was taken for a full blood count, liver function studies, and electrolyte analysis. Because previous studies ${ }^{10}$ had indicated that patient tolerance was better on Sustet, each patient was started on a run-in dose of $500 \mathrm{mg}$ of Sustet at 1000 in addition to their topical therapy and was seen at weekly intervals between 0900 and 1000 in the outpatient department for one to two weeks. If the IOP was not controlled $(22$ $\mathrm{mmHg}$ or less) the dose was increased to a maximum of $500 \mathrm{mg}$ twice daily $(1000$ and 2200 ). With control of the IOP each patient was maintained on this dose throughout the study. Those patients not controlled were withdrawn and started on alternative treatment.

The patients whose IOP was controlled were allocated randomly to either acetazolamide tablets and dummy Sustet or dummy tablets and active Sustet. Each patient was seen weekly, when the IOP was checked and a blood sample taken for plasma acetazolamide estimation by high performance liquid chromatography (HPLC). After four weeks of treatment the patients were admitted for a 24-hour profile of their IOP. The times of the readings were at $0800,0900,1000,1100,1200,1400,1600$, $1800,2200,0400$, and 0800 . Blood was taken simultaneously for plasma acetazolamide estimation. At the end of the study each patient completed a questionnaire about specific side effects during the previous month's treatment. Before discharge each patient was given the alternative systemic formulation and seen again at weekly intervals in the outpatient department for four weeks. They were then readmitted for a second 24-hour profile. On this occasion in addition to their comments on the possible side effects each patient was asked if they had a preference for either period of treatment. Finally the patients completed a third profile of their IOP one week later after cessation of systemic treatment.

\section{Results}

Twenty patients completed the protocol. Of the 35 that were started on the run-in dose of Sustet 11 were withdrawn on account of side effects during this period. The remainder of the patients who were withdrawn did so during the crossover study - two on account of lack of control of IOP, one for personal reasons, and the other because of tinnitus exacerbated by an upper respiratory tract infection. Seventeen patients completed the third inpatient profile of their IOP while not taking acetazolamide. Three could not do so for personal reasons.

Of those patients who completed the crossover study 11 were on topical timolol $0 \cdot 25 \%$ twice daily and the remaining nine were on pilocarpine $2 \%$ or $4 \%$ four times daily. Sixteen were taking $500 \mathrm{mg}$ acetazolamide at night, one was on 500 


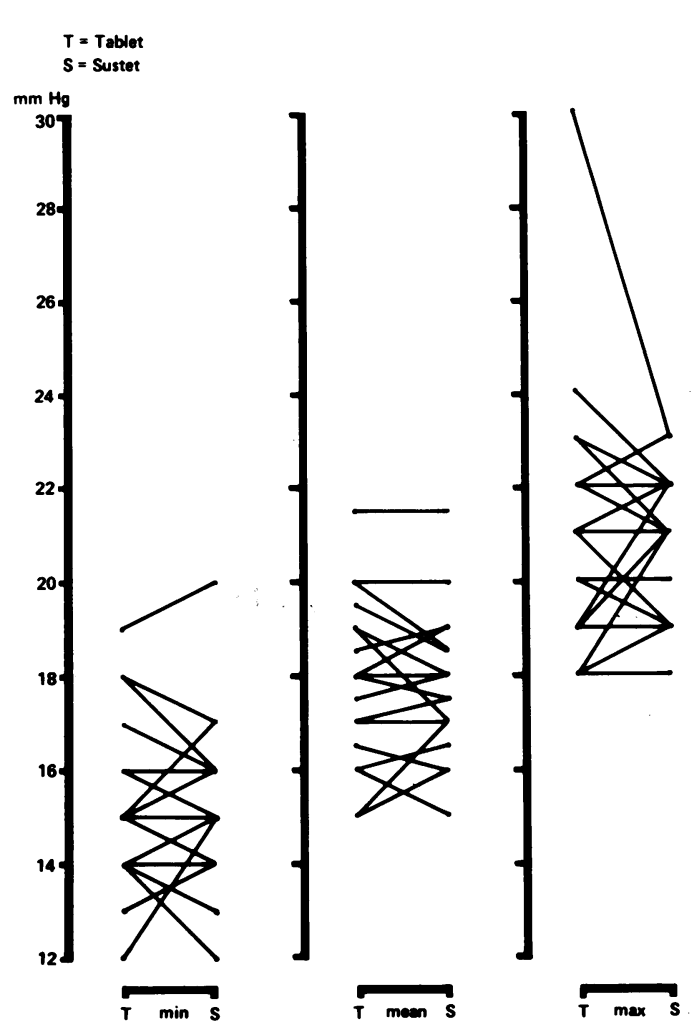

Figure 1: A comparison of the maximum, mean, and minimum IOP ( $\mathrm{mmHg}$ ) in the worse eyes of 20 patients who had been on acetazolamide tablets and Sustets.

mg in the morning, and three patients were taking $500 \mathrm{mg}$ twice daily. Of the seventeen patients who completed the post withdrawal profile (control) 10 were on timolol and the remainder on pilocarpine.

The results of the 24-hour profiles of IOP in the worse eyes of those patients who completed the protocol showed that the mean, minimum, and maximum IOP were indistinguishable between both formulations (Fig 1). Furthermore the diurnal curves for the mean IOP were also similar (Fig 2). Nonetheless it is apparent from the post-withdrawal profile that acetazolamide did have an effect, as there was a considerable rise in IOP. Interestingly the difference between
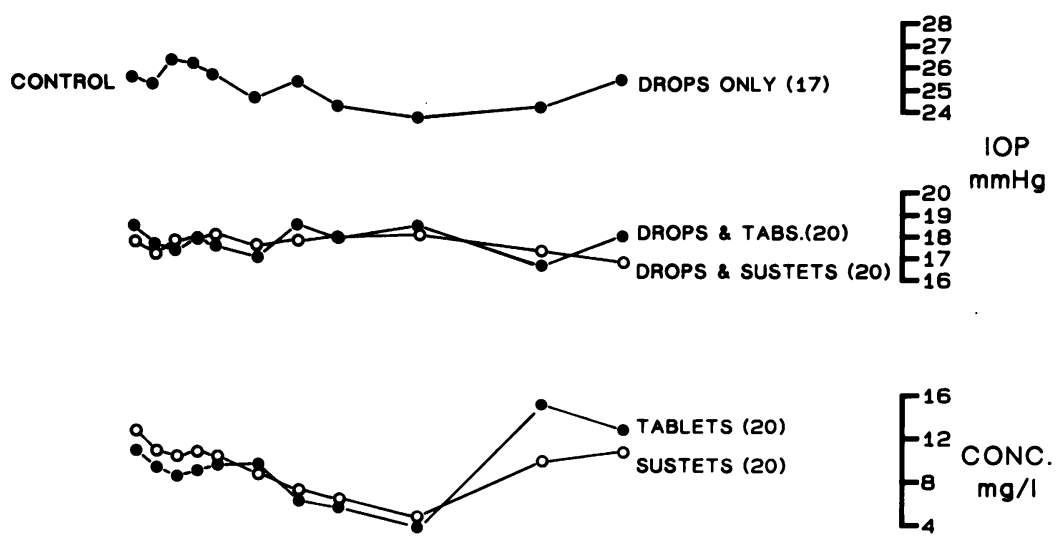

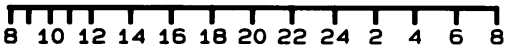

TIME (HOURS).

Figure 2: The mean diumal profile on and off treatment with acetazolamide. The upper half of the profile refers to the IOP ( $\mathrm{mmHg})$ both on and off (control) systemic treatment. The lower half refers to the concentration of acetazolamide (mg/l) measured over the same period (tablets and Sustets). the mean IOP of those patients on timolol alone $(25.31 \mathrm{mmHg}$, SD 0.7$)$ and those on pilocarpine $(25.42 \mathrm{mmHg}$, SD 1.43$)$ was not significant (Fig 3).

It was noteworthy that there was little difference between the mean, minimum, and maximum plasma concentrations of both formulations (Fig 4) during the inpatient profiles. This could have been due to the fact that most patients had an evening dose and the peak concentration was therefore missed. Nevertheless the range on acetazolamide tablets $(15.5 \mathrm{mg} / \mathrm{l}, \mathrm{SD} 4 \cdot 7)$ was greater than on Sustets $(11.6 \mathrm{mg} / \mathrm{l}, \mathrm{SD} 4.9)$. It was significant that there was no discernible relationship between the diurnal plasma concentration of acetazolamide (tablets or Sustets) and the IOP (Figs 2, 3). This is clearly demonstrated by the fact that there was no relationship between the mean plasma concentration and the highest IOP in the worse eyes (Fig 5).

The results of the questionnaire on specified side effects and their severity are presented in Table I. The number of patients reporting particular side effects on both formulations did not differ greatly. Furthermore there was no change in the blood counts of any patient. There was, however, a small reduction in the serum potassium during the first month but this was not sustained. The serum creatinine and bilirubin rose slightly during the same period but this tendency was not maintained for the remainder of the study.

At the end of the crossover study each patient was asked if he or she had a preference for either course of treatment. Of the 17 patients on once daily treatment one preferred the tablets, another the Sustets, and the rest had no preference. Of the three patients on twice daily treatment two preferred the Sustet, one found it easier to swallow, and the second felt better. The third patient had no preference.

\section{Discussion}

Recent studies on acetazolamide have concentrated on the short term effects on IOP. ${ }^{12}$ In particular Friedland $e t$ al " showed that a dose of $63 \mathrm{mg}$ of acetazolamide produced a drop of IOP which higher doses did not increase significantly in a group of ocular hypertensives. This does gave a plasma value of $4-5 \mathrm{mg} / \mathrm{l}$, which is calculated to give $99 \%$ inhibition of carbonic anhydrase in the ciliary epithelium. In our study the mean plasma concentration on acetazolamide tablets was $8.6 \mathrm{mg} / \mathrm{l}(\mathrm{SD} 2.8)$ and on Sustets was $9.7 \mathrm{mg} / \mathrm{l}$ (SD 3.8). This could account for the satisfactory control of IOP in this group of patients, but the topical treatment may have had an overriding influence. However, the withdrawal of acetazolamide produced a significant rise in IOP (Fig 2). Perhaps a future study might look at the plasma levels in those patients whose IOP was not controlled satisfactorily on a given regimen.

Berson et $a l^{14}$ showed that there was no significant difference in the ability of $500 \mathrm{mg}$ Sustet given twice daily to lower the IOP in comparison with $250 \mathrm{mg}$ of acetazolamide tablet four times a day in a group of glaucomatous patients uncontrolled on one or more topical 

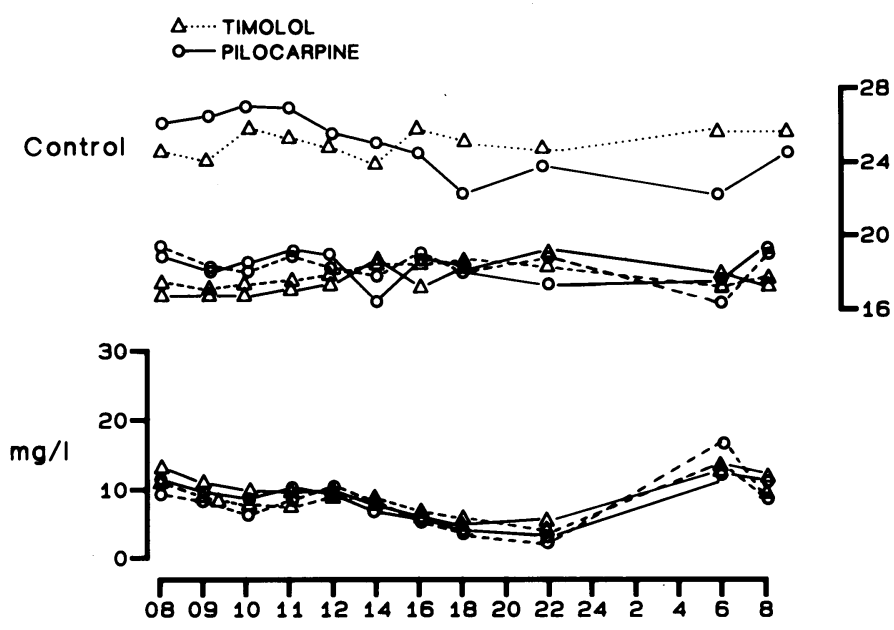

Hours

Figure 3: The diurnal profiles for the patients on timolol $(\triangle)$ and pilocarpine $(\bigcirc)$. The upper half refers to the mean IOP ( $\mathrm{mmHg}$ ) on and off (control) acetazolamide in each group. The lower half refers to the mean concentration of acetazolamide $(\mathrm{mg} / \mathrm{l})$ measured over the same period (tablets or Sustets).

agents. However, this was another short term study. In our study the mean IOP off acetazolamide was $25 \cdot 1 \mathrm{mmHg}$ (SD 1.4). It is interesting to observe that the mean IOP on both preparations was similar $-18 \mathrm{mmHg}(\mathrm{SD} 1 \cdot 8)$ for tablets and $18.1 \mathrm{mmHg}(\mathrm{SD} \mathrm{1.6})$ for Sustets. Since the majority of patients were on an evening dose of acetazolamide only, once daily treatment would appear to be adequate to control IOP. Furthermore the mean plasma level on acetazolamide tablets and Sustets was well above the suggested

TABLE I Number of patients reporting side effects from acetazolamide on the cross-over study with the degree of severity on both formulations

\begin{tabular}{|c|c|c|c|c|c|c|}
\hline & \multicolumn{3}{|c|}{ Tablet } & \multicolumn{3}{|l|}{ Sustet } \\
\hline & Mild & Moderate & Severe & Mild & Moderate & Severe \\
\hline Malaise & 3 & & & 3 & & \\
\hline Nausea & 1 & & & 2 & & \\
\hline Weakness & 3 & & & 3 & & \\
\hline Depression & 1 & & & 0 & & \\
\hline Paraesthesiae & 10 & 8 & 1 & 12 & 7 & \\
\hline Alteration of taste & 2 & & & 1 & & \\
\hline Diarrhoea & 3 & & & 4 & & \\
\hline Anorexia & 4 & & & 4 & & \\
\hline Drowsiness & 6 & & & 7 & & \\
\hline Diuresis (short) & 4 & & & 4 & & \\
\hline Constipation & 2 & & & 0 & & \\
\hline
\end{tabular}

$$
\text { IOP }
$$

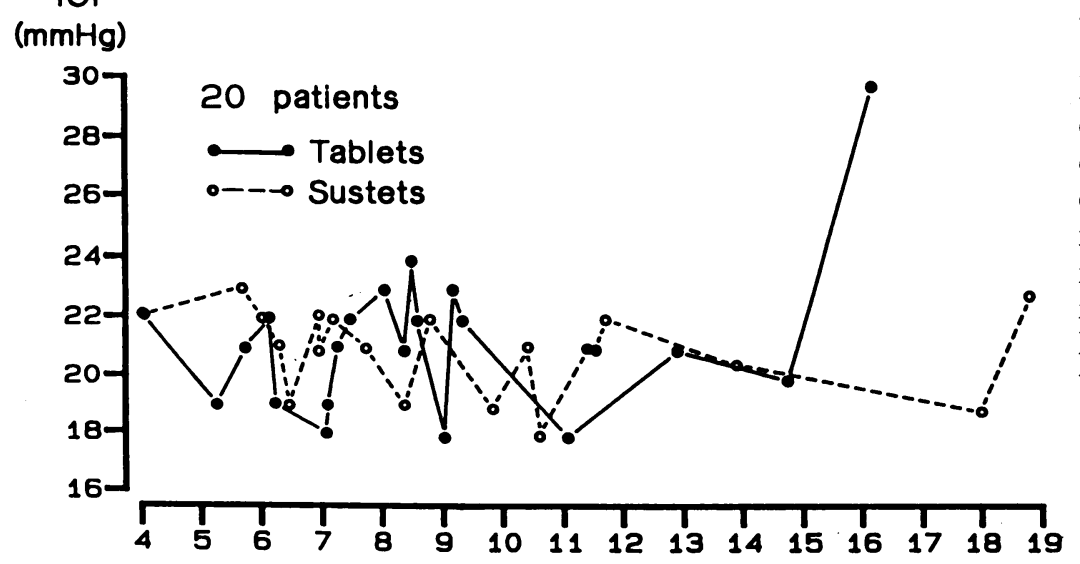

Acetazolamide mean conc. (mg/l)

Figure 5: The mean concentration (mg/l) of acetazolamide in 20 patients versus the maximum recorded IOP $(\mathrm{mmHg})$ in the worse eyes.
ACETAZOLAMIDE CONCENTRATIONS

$T=$ Tablet
$S=$ Sustet
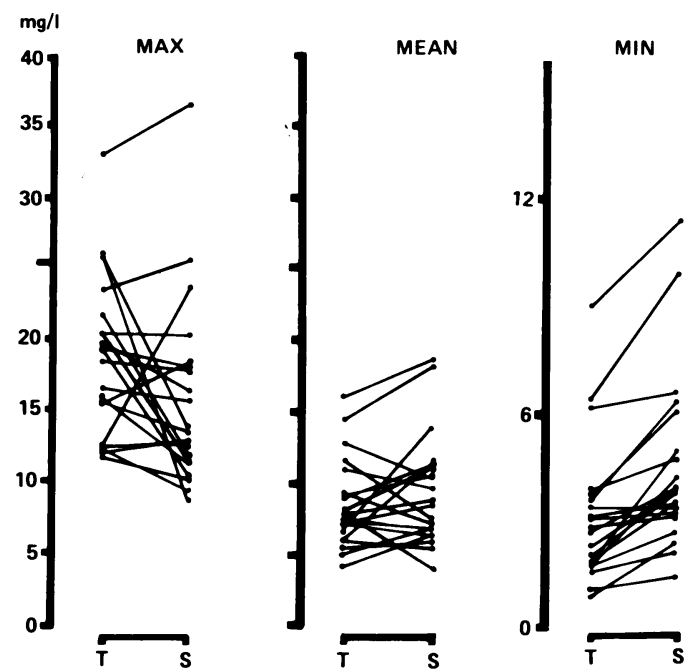

Figure 4: A comparison of the maximum, mean, and minimum acetazolamide concentration $(\mathrm{mg} / \mathrm{l})$ in the 20 patients who had been on tablets and Sustets.

ideal plasma level of $5 \mathrm{mg} / 1 .{ }^{15}$ This suggests perhaps that an even smaller dose of acetazolamide tablets $(250 \mathrm{mg})$ should be tried initially for long-term treatment, whereas a higher dose (500 $\mathrm{mg}$ ) has been shown to produce the highest reduction in IOP in the short term. ${ }^{16}$ From the post-withdrawal profile (control, Fig 3 ) there is a trend for the diurnal curve on timolol to be smoother than on pilocarpine, confirming similar findings of $\mathrm{McCl}$ ure and Vogel $^{17}$ and Hass and Drance. ${ }^{18}$

During the run-in period of this study patients were started on $500 \mathrm{mg}$ of Sustet in the morning to counteract the diurnal rise in IOP. However, 11 patients were withdrawn owing to side effects. In an attempt to improve recruitment an evening dose was tried. This reduced the incidence and severity of the side effects considerably. When the code of the trial was broken, it was surprising to find there was no significant difference in the number of patients reporting specified side effects on the two formulations of acetazolamide. This finding lends support for an evening dose schedule and perhaps to the common belief that the subjective side effects relate to the peak concentration, especially with long-term use.

In conclusion, most patients in this study, who had primary open-angle glaucoma uncontrolled on single topical treatment, were adequately controlled on the addition of $500 \mathrm{mg}(2 \times 250 \mathrm{mg})$ of acetazolamide tablets or one Sustet $(500 \mathrm{mg})$ at night. The severity of side effects was also reduced. Thus an evening dose of acetazolamide in conjunction with topical therapy may well have a beneficial effect on compliance in the treatment of open-angle glaucoma.

1 Becker B. Decrease in intraocular pressure in man by carbonic anhydrase inhibitor, Diamox. Am f Ophthalmol 1954; 37: 13 .

2 Drance SM, Carr F. Effect of sustained-release Diamox on the intraocular pressure in man. $\mathrm{Br} \mathcal{F}$ Ophthalmol 1961; 45: 695-8.

3 Mestre C, Galin M, McLean J. Evaluation of sustained-release acetazolamide. Br f Ophthalmol 1963; 47: 31-5.

4 Garner L, Franklin CE, Ferweda J. Advantages of sustainedrelease therapy with acetazolamide in glaucoma. $A m \mathcal{F}$ Ophthalmol 1963; 55: 323-7. 
5 Becker B. Longterm acetazolamide (Diamox) administration in therapy of glaucomas. Arch Ophthalmol 1955; 54: 187-92.

6 Kupfer K, Lawrence C, Linner E. Longterm administration of acetazolamide (Diamox) in the treatment of glaucoma. $\mathrm{Am}$ f Ophthalmol 1955; 40: 673-80.

7 de Carvalho CA, Lawrence C, Stone H. Acetazolamide (Diamox) therapy in chronic glaucoma. Arch Ophthalmo 1958; 59: 840-9.

8 Garrison 1 Roth comparison of three carbonic anhydrase inhibitors. Trans Pacific Coast Oto-ophthalmol Soc 1967; 48: 137-45.

9 Lichter P, Newman L, Wheeler N, Beall O. Patient tolerance to carbonic anhydrase inhibitors. Am $\mathcal{F}$ Ophthalmol 1978; 85: 495-502.

10 Berson F, Epstein D. Carbonic anhydrase inhibitors: management of side effects. Perspect Ophthalmol 1980; 4: 91-5.

11 Friedland B, Mallonee J, Anderson D. Short-term dose response characteristics of acetazolamide in man. Arch Ophthalmol 1977; 95: 1809-12.

12 Foster TS, Kielar RA, Dipiro JT. Clinical and bioavailability studies of two formulations of acetazolamide. A scientific exhibit. Lederle Laboratories, Litho, USA, 1982.

13 Joyce PW. Acetazolamide in primary open angle glaucoma. University of Manchester, 1987: MSc thesis.

14 Berson F, Epstein D, Grant W, Hutchinson T, Dobbs P. Acetazolamide dosage forms in the treatment of glaucoma. Arch Ophthalmol 1980; 98: 1051-4.

15 Wistrand PJ. The use of carbonic anhydrase inhibitors in ophthalmology and clinical medicine. Ann NY Acad Sci 1984; 424: 609-19.

16 Lichter P, Musch D, Medzihradsky F, Standardi L. Intraocular pressure effects of carbonic anhydrase inhibitors in primary open angle glaucoma. Am $\mathcal{F}$ Ophthalmol 1989; 107 $11-7$.

17 Maclure GM, Vogel R. The effect on diurnal control of IOP of co-administration of pilocarpine and Timoptol: an interim report. Res Clin Forums 1985; 7: 85-91.

18 Hass I, Drance SM. Comparison between pilocarpine and timolol on diurnal pressure in open angle glaucoma. Arch Ophthalmol 1980; 98: 480-1. 